\title{
Home blood pressure and cardiovascular risk in treated hypertensive patients: the prognostic value of the first and second measurements and the difference between them in the HONEST study
}

\author{
Ikuo Saito ${ }^{1}$, Kazuomi Kario ${ }^{2}$, Toshio Kushiro ${ }^{3}$, Satoshi Teramukai ${ }^{4}$, Mai Yaginuma ${ }^{5}$, Yoshihiro Mori ${ }^{5}$, \\ Yasuyuki Okuda ${ }^{5}$ and Kazuyuki Shimada ${ }^{6}$
}

Hypertension guidelines recommend using the average of two home blood pressure (HBP) measurements obtained on one occasion to monitor blood pressure. We studied the prognostic value of the first and second measurements or their average value during the follow-up period, as well as the relationships among the difference between the first and second HBP measurements and the prognosis using data from the HONEST (HBP measurement with Olmesartan-Naive patients to Establish Standard Target blood pressure) study. During the mean 2.02 years follow-up, 280 patients had cardiovascular events. Hazard ratios (HRs) for cardiovascular events for each $1 \mathrm{~mm} \mathrm{Hg}$ increase in the first, second and averaged morning home systolic blood pressure (MHSBP) were similar. Hazards were significantly higher in patients with a large difference between the first and second MHSBP ( $\triangle$ MHSBP) of $<-5 \mathrm{~mm} \mathrm{Hg}$ (HR: 2.12) or $\geqslant 5 \mathrm{~mm} \mathrm{Hg}$ (HR: 1.44) compared with those with a small $\triangle M H S B P$ of $\geqslant-5$ to $<5 \mathrm{~mm} \mathrm{Hg}$ using the Cox proportional hazards model adjusted for the averaged MHSBP during the follow-up and other risk factors. Hazards in patients with an averaged MHSBP $\geqslant 145 \mathrm{mmHg}$ and a small $\triangle$ MHSBP (HR: 3.11), those with an averaged MHSBP $\geqslant 125$ to $<145 \mathrm{~mm} \mathrm{Hg}$ and a large $\triangle$ MHSBP (HR: 1.91) and those with an averaged MHSBP $\geqslant 145 \mathrm{~mm} \mathrm{Hg}$ and a large $\triangle$ MHSBP (HR: 4.63) were higher compared with those with an averaged MHSBP $<125 \mathrm{~mm} \mathrm{Hg}$ and a small $\triangle$ MHSBP. In conclusion, the first, second and averaged MHSBP measurements have similar prognostic values. Prognosis is worse for patients with a large $\triangle$ MHSBP. In clinical practice, it would be prudent to measure the HBP two times and use the average HBP of two measurements obtained on one occasion with particular attention to patients with a large $\triangle$ MHSBP.

Hypertension Research (2016) 39, 857-862; doi:10.1038/hr.2016.99; published online 4 August 2016

Keywords: cardiovascular risk; guideline; home blood pressure monitoring; prognosis

\section{INTRODUCTION}

Home blood pressure (HBP) monitoring is useful in the diagnosis and treatment of hypertension and has been shown to have a greater prognostic value than clinic blood pressure (CBP) monitoring. ${ }^{1-7}$ The Japanese Society of Hypertension Guidelines for the Management of Hypertension (JSH 2014) ${ }^{8}$ and the European Society of HypertensionEuropean Society of Cardiology guidelines ${ }^{9}$ recommend using the average of two HBP measurements obtained on one occasion to determine a person's blood pressure. The prognostic values of the first and second HBP measurements obtained on one occasion at baseline have been investigated in the Finn-Home study ${ }^{10}$ and the Didima study. ${ }^{11}$ However, the prognostic value of the first, second and the averaged HBP during the follow-up period is unclear. Moreover, the second measurement of systolic HBP is often lower compared with the first, ${ }^{12,13}$ but the prognostic value of the difference between these two measurements has yet to be clarified.

The HBP measurement with Olmesartan-Naive patients to Establish Standard Target blood pressure (HONEST) study is a large-scale, prospective, observational study performed to investigate the relationship between morning HBP and CBP during the follow-up period and the risk of cardiovascular events in $>20000$ Japanese patients with hypertension. ${ }^{12,14}$ A primary analysis of the data from the HONEST study has shown that cardiovascular risk is increased in patients with a morning home systolic blood pressure (MHSBP) of $\geqslant 145 \mathrm{~mm} \mathrm{Hg}$, even when CBP is controlled. ${ }^{1}$ This finding emphasizes the importance of HBP monitoring in clinical practice.

In the present analysis, we used the data from the HONEST study to determine the prognostic value of the first and second HBP

${ }^{1}$ Keio University Health Center, Yokohama, Japan; ${ }^{2}$ Division of Cardiovascular Medicine, Department of Medicine, Jichi Medical University School of Medicine, Shimotsuke, Japan; ${ }^{3}$ The Life Planning Center Foundation, Tokyo, Japan; ${ }^{4}$ Department of Biostatistics, Graduate School of Medical Science, Kyoto Prefectural University of Medicine, Kyoto, Japan; ${ }^{5}$ Daiichi Sankyo, Tokyo, Japan and ${ }^{6}$ Shin-Oyama City Hospital, Oyama, Japan

Correspondence: Professor I Saito, Keio University Health Center, 4-1-1 Hiyoshi, Kohoku-ku, Yokohama, Kanagawa 223-8521, Japan.

E-mail: saitoikuokeiouniv@gmail.com

Received 10 March 2016; revised 16 June 2016; accepted 18 June 2016; published online 4 August 2016 
measurements obtained on one occasion, and their average value, in patients receiving treatment for hypertension. We also investigated the relationship of the difference between the first and second MHSBP ( $\triangle \mathrm{MHSBP})$ and the patient's prognosis. We used measurements of MHSBP because it is the strongest indicator of common cardiovascular events. ${ }^{15}$

\section{METHODS}

\section{Study design}

HONEST is a large-scale, prospective, observational study with a 2-year followup period. The study population comprised patients receiving olmesartan-based treatment for hypertension. The study is registered at the UMIN Clinical Trials Registry (http://www.umin.ac.jp/ctr/index.htm) with the unique trial number of UMIN000002567.

The study protocol was approved by the Ethical Committee of Daiichi Sankyo and the research ethics committees of the participating institutions, at their discretion. The study was approved by the Ministry of Health, Labor and Welfare of Japan before it began and conforms to the country's pharmaceutical affairs laws. In compliance with Good Post-marketing Study Practices in Japan, the study was performed at registered medical institutions.

The aims and the protocol of the HONEST study have been reported previously. ${ }^{12}$ Olmesartan-naive patients with essential hypertension who had their morning HBP recorded for $\geqslant 2$ days and their CBP recorded for $\geqslant 1$ day in the 28 days before starting olmesartan were eligible to participate. The patients were enrolled in the study after providing written informed consent and being prescribed olmesartan. Those who had had a cardiovascular event in the previous 6 months were excluded, as well as those with a planned cardiovascular intervention or serious hepatic or renal dysfunction. The primary end point was a composite of cerebrovascular events (cerebral infarction, intracerebral hemorrhage, subarachnoid hemorrhage and unclassified stroke), cardiac events (myocardial infarction and coronary revascularization procedures for angina pectoris) and sudden death. Follow-up was maintained for patients who discontinued treatment with olmesartan.

\section{HBP measurements}

Patients who already possessed a cuff oscillometric device were enrolled in the study. All such devices available in Japan have been validated and approved by the Ministry of Health, Labor and Welfare. The devices also comply with the Association for the Advancement of Medical Instrumentation ${ }^{16}$ or European standards. ${ }^{17}$ At the time that informed consent was obtained, the patients were asked to measure their HBP two times in the morning (within $1 \mathrm{~h}$ of waking, after urination, before taking any morning medications, before breakfast and after 1-2 min of rest in a sitting position) and two times at bedtime (after 1-2 min of rest in a sitting position), according to the Japanese Society of Hypertension Guidelines for the Management of Hypertension (JSH 2009). ${ }^{18}$ Participating patients in the present study received a pocket diary to record their HBP. Physicians instructed the patients to report their HBP obtained on the prespecified measurement day and the day before (when this was not possible, on 2 days in the week before or after the prespecified measurement day). Details of the timing and frequency of measurements were indicated in the informed consent form and the pocket diary.

During the follow-up, the HBP was measured at 1, 4 and 16 weeks and at 6, 12, 18 and 24 months. In principle, patients reported their HBP that were measured two times in the morning and two times at bedtime on two different days for each of these measurement points. When there were several HBP measurements at each measurement time, the physicians gave priority to any measurements reported for 2 days, for which both the first and second measurements of the morning and evening were available. In addition, measurements taken on days close to the prespecified measurement day were also used; however, when the differences in days from the prespecified measurement day were the same, the measurement of the day before the other was used. For each HBP variable, namely the first, second and averaged MHSBP measurements, and $\triangle$ MHSBP, the mean value over 2 days (provided the necessary data were available) was calculated for each measurement point. Moreover, the mean HBP values for the maximum of the seven measured time points (1, 4 and 16 weeks and 6, 12, 18 and 24 months) during the follow-up period were further averaged and used in the analyses of hazard ratios (HRs).

\section{Statistical analysis}

The analysis set comprised eligible patients who had received olmesartan at least once.

To determine their prognostic value for cardiovascular events, HRs for each $1 \mathrm{mmHg}$ increase in the first, second and averaged MHSBP during the followup period were estimated using the Cox proportional hazards model, which was adjusted for sex, age, family history of cardiovascular disease, dyslipidemia, diabetes mellitus, chronic kidney disease, history of cardiovascular disease and smoking status. Furthermore, multivariate generalized additive models adjusted for risk factors as mentioned above with a spline function of three degrees of freedom were used to investigate potential nonlinear relationships between the $\triangle$ MHSBP during the follow-up as continuous variables as well as the incidence of cardiovascular events.

Subsequently, to investigate the relationship between $\triangle$ MHSBP and prognosis, we used a multivariate Cox proportional hazards model adjusted for MHSBP during the follow-up period and other risk factors as mentioned above (i.e., sex, age, family history of cardiovascular disease, dyslipidemia, diabetes mellitus, chronic kidney disease, history of cardiovascular disease and smoking status) to compare hazards for the three groups of patients stratified by $\triangle$ MHSBP: $<-5, \geqslant-5$ to $<5$ and $\geqslant 5 \mathrm{~mm} \mathrm{Hg}$.

To further investigate the relationships between $\triangle$ MHSBP and the average value of these two measurements, we compared hazards for the six groups of patients classified first by $\triangle$ MHSBP (large difference, $<-5$ or $\geqslant 5 \mathrm{~mm} \mathrm{Hg}$; small difference, $\geqslant-5$ to $<5 \mathrm{~mm} \mathrm{Hg}$ ) and second by the averaged MHSBP $(<125, \geqslant 125$ to $<145$ and $\geqslant 145 \mathrm{~mm} \mathrm{Hg})$ adjusted for risk factors as mentioned above (i.e., sex, age, family history of cardiovascular disease, dyslipidemia, diabetes mellitus, chronic kidney disease, history of cardiovascular disease and smoking status). The cutoff values for MHSBP of 125 and $145 \mathrm{~mm} \mathrm{Hg}$ were chosen based on the findings in the main HONEST study, ${ }^{1}$ in which cardiovascular risk significantly increased at an MHSBP of $\geqslant 145 \mathrm{~mm} \mathrm{Hg}$ and was at a minimum at an MHSBP of $124 \mathrm{~mm} \mathrm{Hg}$ during the follow-up period.

All statistical tests were two-sided, using a significance level of $P<0.05$. SAS release 9.2 software (SAS Institute, Cary, NC, USA) was used for all statistical analyses.

\section{RESULTS}

Data were collected from 22298 of the 22373 patients enrolled in the study. After eliminating data from patients who met the exclusion criteria, data from 21591 patients were included in the analysis. The mean age of the patients enrolled in the study was $64.9 \pm 11.9$ years; $51 \%$ of the patients were female; $50 \%$ of the patients received prior antihypertensive treatment; $45 \%$ of patients had dyslipidemia; $21 \%$ of patients had diabetes mellitus; $20 \%$ of patients had chronic kidney disease; and $11 \%$ of patients had a history of cerebral or cardiovascular disease. The mean follow-up period was 2.02 years.

The first, second and averaged MHSBP were $151.6 \pm 16.4 \mathrm{~mm} \mathrm{Hg}$ $(n=21587), 150.1 \pm 16.2 \mathrm{~mm} \mathrm{Hg}(n=7730)$ and $151.2 \pm 16.3 \mathrm{~mm} \mathrm{Hg}$ $(n=21588)$, respectively, at baseline, and $135.8 \pm 10.9 \mathrm{~mm} \mathrm{Hg}(n=20$ 999), $\quad 133.8 \pm 11.1 \mathrm{~mm} \mathrm{Hg} \quad(n=18 \quad 251)$ and $135.2 \pm 10.8 \mathrm{~mm} \mathrm{Hg}$ $(n=21001)$, respectively, during the follow-up period. In patients whose first and second MHSBP values were both available $(n=18249)$, the first, second and averaged MHSBP during the follow-up period were $135.7 \pm 10.8,133.8 \pm 11.1$ and $135.1 \pm 10.6 \mathrm{~mm} \mathrm{Hg}$, respectively. In the present analysis of the average value, in accordance with JSH $2014,{ }^{8}$ we used the average of the two HBP measurements obtained on each occasion, if available; if these measurements were not available, a single measurement was used. A total of 280 patients reached the primary end point (incidence, 6.46/1000 patient-years).

Hazards for cardiovascular events increased significantly with each $1 \mathrm{mmHg}$ increase in the first, second and averaged MHSBP during the 


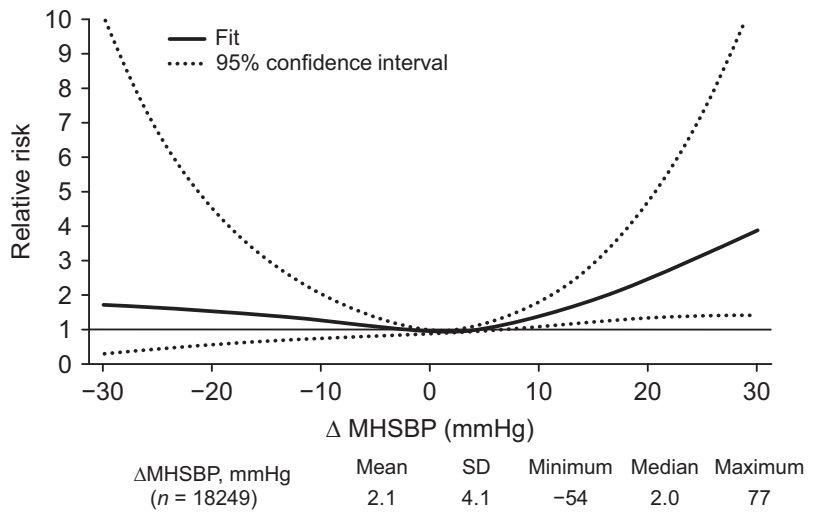

Figure 1 The relationship between $\triangle$ MHSBP during the follow-up period and cardiovascular risk (continuous evaluation by spline regression analysis, adjusted for sex, age, family history of cardiovascular disease, dyslipidemia, diabetes mellitus, chronic kidney disease, history of cardiovascular disease and smoking status). $\triangle \mathrm{MHSBP}$, the difference between the first and second measurements of the home systolic blood pressure taken in the morning.

follow-up period; the HR (95\% confidence interval (95\% CI)) for each was 1.039 (1.028-1.049), 1.038 (1.027-1.049) and 1.039 (1.029-1.049), respectively.

The analysis using a spline regression model showed that relative risk increased proportionally with $\triangle \mathrm{MHSBP}$ (Figure 1). A mean of 4.6 individual calculations (maximum, 7) using data from separate measurement points was used to generate the $\triangle \mathrm{MHSBP}$ value for each patient.

Table 1 shows the baseline characteristics of the three groups of patients classified by $\triangle$ MHSBP; mean age was significantly higher in the two groups of patients with a large difference $(<-5$ and $\geqslant 5 \mathrm{~mm} \mathrm{Hg})$ compared with the group with a small difference $(\geqslant-5$ to $<5 \mathrm{~mm} \mathrm{Hg}$ ). The patients with $\Delta$ MHSBP of $<-5 \mathrm{~mm} \mathrm{Hg}$ were significantly more likely to have had a history of cerebrovascular or cardiovascular disease or diabetes mellitus. The patients with $\Delta$ MHSBP of $\geqslant 5 \mathrm{~mm} \mathrm{Hg}$ were significantly more likely to be women, to have had dyslipidemia or previous antihypertensive treatment and to have been receiving concomitant drugs at study entry and were less likely to be current smokers and regular alcohol drinkers.

The averaged MHSBP of patients with a $\triangle$ MHSBP of $<-5, \geqslant-5$ to $<5$ and $\geqslant 5 \mathrm{~mm} \mathrm{Hg}$ were $151.0 \pm 16.9, \quad 150.6 \pm 16.1$ and $151.9 \pm 16.4 \mathrm{~mm} \mathrm{Hg}$, respectively, at baseline, and $136.9 \pm 13.5$, $134.7 \pm 10.3$ and $136.3 \pm 11.4 \mathrm{~mm} \mathrm{Hg}$, respectively, during the follow-up period.

Hazards (adjusted for cardiovascular risk factors and the averaged MHSBP during the follow-up period) were significantly increased for patients with a large $\triangle$ MHSBP compared with for those with a small $\triangle$ MHSBP (Figure 2).

A comparison of the six groups of patients classified by the $\triangle \mathrm{MHSBP}$ and the averaged MHSBP shows significantly increased hazards for patients with an averaged MHSBP of $\geqslant 145 \mathrm{~mm} \mathrm{Hg}$ in the group with a small $\triangle$ MHSBP, and for those with an averaged MHSBP of $\geqslant 125 \mathrm{~mm} \mathrm{Hg}$ in the group with a large $\triangle$ MHSBP compared with that in the group with an averaged MHSBP of $<125 \mathrm{mmHg}$ and a small $\triangle$ MHSBP (Figure 3). With a cutoff value for MHSBP of $135 \mathrm{~mm} \mathrm{Hg}$ (as consistent with the threshold in JSH 2014), the hazards were higher in patients with an averaged MHSBP of $\geqslant 135 \mathrm{~mm} \mathrm{Hg}$ and a small $\triangle$ MHSBP (HR: 1.85 ; 95\% CI: 1.32 $2.61)$ ), those with an averaged MHSBP of $<135 \mathrm{~mm} \mathrm{Hg}$ and a large $\triangle$ MHSBP (HR: 2.17; 95\% CI: 1.35-3.49) and those with an averaged
MHSBP of $\geqslant 135 \mathrm{~mm} \mathrm{Hg}$ and a large $\triangle$ MHSBP (HRs: 2.50; 95\% CI: 1.65-3.80), compared with those with an averaged MHSBP of $<135 \mathrm{~mm} \mathrm{Hg}$ and a small $\triangle$ MHSBP.

\section{DISCUSSION}

The results of the present analysis show that the first and second measurements of MHSBP obtained on one occasion and their average value during the follow-up period have similar prognostic value for the assessment of cardiovascular risk. Prognosis is worse for patients with a large difference between the first and second MHSBP measurements.

Prognostic value for each $1 \mathrm{mmHg}$ increase in each $\mathrm{HBP}$ variable In the HONEST study, as in the Finn-Home study ${ }^{10}$ and the Didima study, ${ }^{11}$ the HBP was measured two times on single occasions. In the HONEST study, the difference between the first and second measurements of MHSBP was small $(\geqslant-5$ to $<5 \mathrm{~mm} \mathrm{Hg})$ in $\sim 75 \%$ of patients, and we found a similar prognostic value between the first and second MHSBP measurements during the follow-up period. In the Finn-Home study, participants from the general population had two HBP measurements taken in both the morning and in the evening for 7 consecutive days at baseline; the relative hazards (95\% CI) for each $1 \mathrm{mmHg}$ increase in the first and second SBP measurement were 1.020 (95\% CI: $1.011-1.028)$ and 1.022 (95\% CI: 1.014-1.031), respectively. ${ }^{10}$ In the Didima study, participants were also enrolled from the general population, and the HBP was measured in the same way for 3 days at baseline; HRs per $1 \mathrm{mmHg}$ increase were similar for the first and second measurements (1.033 and 1.035, respectively), although the first measurement was on average $3.5 \mathrm{~mm} \mathrm{Hg}$ higher compared with the second. ${ }^{11}$

\section{Prognostic value of the difference between the first and second measurements}

In the past few years, the relationship between $\mathrm{BP}$ variability and cardiovascular risk has been the focus of several clinical studies. A morning surge in $\mathrm{BP}$ has been reported to predict cardiovascular events. ${ }^{19}$ Associations between prognosis and circadian variability, ${ }^{20}$ day-to-day variability ${ }^{21,22}$ and visit-to-visit variability have also been reported. $^{23}$

Differences between the first and subsequent BP measurements on one occasion may indicate a type of short-term variability. In the FinnHome study, the s.d. of the difference between the first and second HBP measurements was found to predict cardiovascular events. ${ }^{21}$ However, no study has been performed to investigate the relationship among the actual differences between the first and subsequent HBP measurements and cardiovascular events.

In the present analysis, we considered $\pm 5 \mathrm{~mm} \mathrm{Hg}$ to be the cutoff value for clinically significant changes based on the results from the spline regression model using continuous variables of $\triangle \mathrm{MHSBP}$ during the follow-up period and the incidence of cardiovascular events. We found that, while $\Delta$ MHSBP $\geqslant-5$ to $<5 \mathrm{~mm} \mathrm{Hg}$ had very little effect on cardiovascular risk, the risk increased for an $\triangle$ MHSBP of $<-5$ or $>5 \mathrm{~mm} \mathrm{Hg}$ (Figure 1).

Subsequently, using the cutoff value of $\pm 5 \mathrm{~mm} \mathrm{Hg}$, to compare hazards we classified patients into three groups by the $\triangle$ MHSBP. Consequently, we found that the difference between the first and second HBP measurements obtained on one occasion was a cardiovascular risk factor independent of actual HBP values.

With respect to baseline characteristics, patients with a large $\triangle$ MHSBP $(\triangle \mathrm{MHSBP}<-5$ or $>5 \mathrm{~mm} \mathrm{Hg}$ ) tended to have a higher average age compared with patients with a small difference $(\geqslant-5$ to $<5 \mathrm{~mm} \mathrm{Hg}$ ). 
Table 1 Baseline characteristics of patients in the HONEST study

\begin{tabular}{|c|c|c|c|c|c|}
\hline & \multicolumn{3}{|c|}{$\triangle M H S B P(m m ~ H g)$} & \multicolumn{2}{|c|}{$P$-value ${ }^{\text {a }}$ vs. $\triangle M H S B P \geqslant-5$ to $<5 \mathrm{~mm} \mathrm{Hg}$ ) } \\
\hline & $<-5$ & $\geqslant-5$ to $<5$ & $\geqslant 5$ & $<-5 \mathrm{~mm} \mathrm{Hg}$ & $\geqslant 5 \mathrm{~mm} \mathrm{Hg}$ \\
\hline Women & $172(48.3)$ & $6983(48.9)$ & 2097 (57.9) & 0.82 & $<0.0001^{\mathrm{b}}$ \\
\hline Mean age (years) & $66.3 \pm 12.0$ & $64.4 \pm 11.9$ & $66.3 \pm 11.1$ & $0.004^{b}$ & $<0.0001^{\mathrm{b}}$ \\
\hline Body mass index $\left(\mathrm{kg} \mathrm{m}^{-2}\right)$ & $24.4 \pm 4.0$ & $24.3 \pm 3.6$ & $24.2 \pm 3.7$ & 0.67 & 0.46 \\
\hline \multicolumn{6}{|l|}{ Disease history } \\
\hline Cerebro- or cardiovascular disease & $61(17.1)$ & $1426(10.0)$ & $385(10.6)$ & $<0.0001^{\mathrm{b}}$ & 0.24 \\
\hline Cerebrovascular disease & $41(11.5)$ & $862(6.0)$ & $244(6.7)$ & $<0.0001^{\mathrm{b}}$ & 0.12 \\
\hline Cardiovascular disease & $25(7.0)$ & $651(4.6)$ & $165(4.6)$ & 0.03 & 1.00 \\
\hline Regular alcohol drinkers & $59(16.6)$ & $2343(16.4)$ & $549(15.1)$ & 0.08 & $<0.0001^{\mathrm{b}}$ \\
\hline Previous antihypertensive drug use & $185(52.0)$ & $7206(50.5)$ & $1941(53.6)$ & 0.58 & $0.001^{\mathrm{b}}$ \\
\hline Antihypertensive drug used at study entry (excluding olmesartan) & $157(44.1)$ & $5519(38.7)$ & $1509(41.6)$ & 0.04 & $0.001^{\mathrm{b}}$ \\
\hline
\end{tabular}

Abbreviations: HBP, home blood pressure; HONEST, HBP measurement with Olmesartan-Naive patients to Establish Standard Target blood pressure; $\triangle \mathrm{MHSBP}$, the difference between the first and second measurements of morning home systolic blood pressure during the follow-up period.

a $\chi^{2}$ Test for categorical data and $t$-test for continuous data. Tested for other than missing cases.

bSignificant result (significant level: $<0.05 / 3$ by Bonferroni correction).

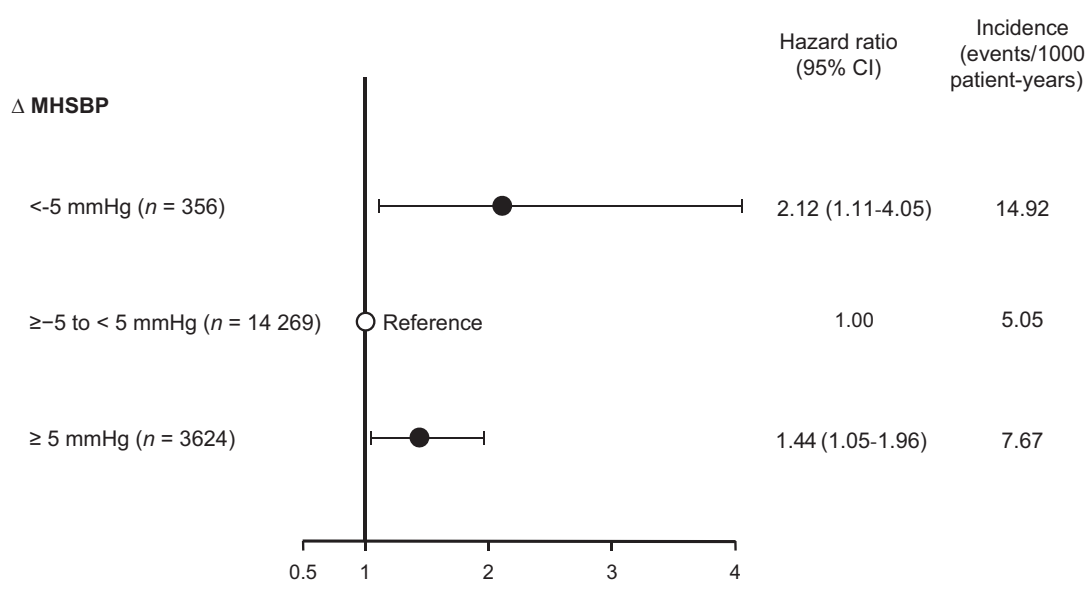

Figure 2 The relationship between $\triangle \mathrm{MHSBP}$ during the follow-up period and cardiovascular risk (reference, $\Delta \mathrm{MHSBP} \geqslant-5$ to $<5 \mathrm{~mm} \mathrm{Hg}$ ). The hazard ratio for cardiovascular events was adjusted for sex, age, family history of cardiovascular disease, dyslipidemia, diabetes mellitus, chronic kidney disease, history of cardiovascular disease, smoking status and the averaged MHSBP during the follow-up period. Cl, confidence interval; $\triangle \mathrm{MHSBP}$, the difference between the first and second measurements of the home systolic blood pressure taken in the morning.

In elderly patients, whose blood vessel elasticity is often reduced by arteriosclerosis, increased BP variability is an independent predictor of cardiovascular events. ${ }^{24}$ Elderly patients also often have a history of various diseases and complications, which may also contribute to their overall high risk of cardiovascular disease. The present analysis was adjusted for sex, age, history of cardiovascular diseases, smoking status, risk complications and, most importantly, the mean MHSBP during the follow-up period, which is the greatest cardiovascular risk factor. Cardiovascular risk was significantly increased in patients with a large $\triangle$ MHSBP, but was independent of actual MHSBP. We also conducted analyses of evening home SBP by using a spline regression model and a Cox regression model, and similar results were observed (data not shown).

A subanalysis of the J-HOP study enrolling 4149 Japanese patients with cardiovascular risk factors investigated the relationship among the differences between the first and second or third HBP measurements and the levels of various indicators of target organ damage. ${ }^{25}$ In that study, patients whose second and third measurements were higher compared with the first measurement were more likely to have diabetes or impaired glucose tolerance, had a higher B-type natriuretic peptide and N-terminal pro-B-type natriuretic peptide levels and had a lower estimated glomerular filtration rate compared with those in the 


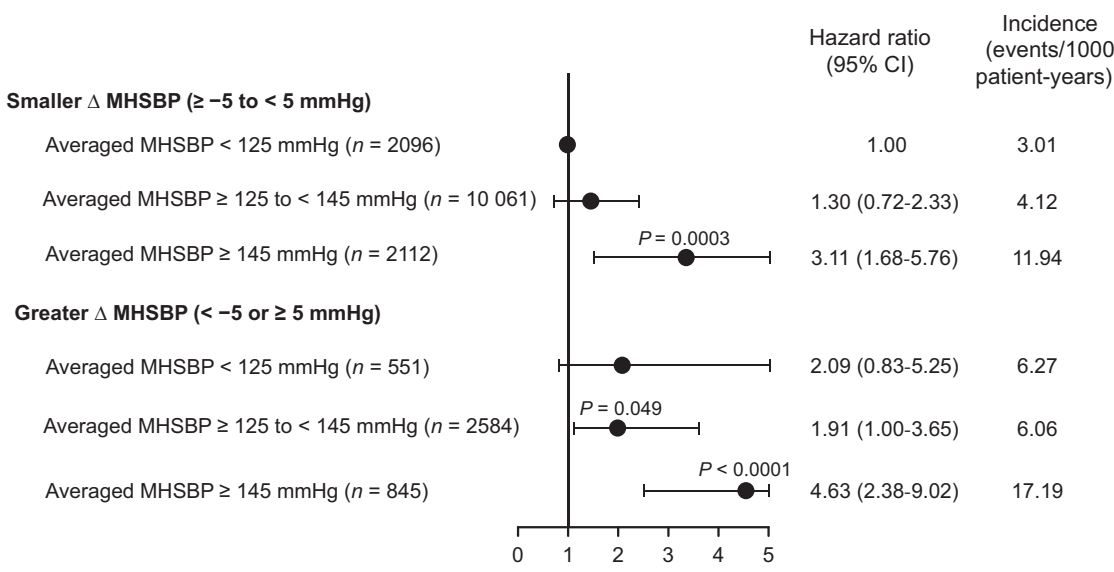

Figure 3 Cardiovascular risk stratified by $\triangle$ MHSBP during the follow-up period and the averaged MHSBP. The hazard ratio for cardiovascular events was adjusted for sex, age, family history of cardiovascular disease, dyslipidemia, diabetes mellitus, chronic kidney disease, history of cardiovascular disease and smoking status. $\triangle \mathrm{MHSBP}$, the difference between the first and second measurements of the home systolic blood pressure taken in the morning.

reference group (quintile median). Moreover, patients with the greatest decreases in subsequent measurements also had higher $\mathrm{N}$-terminal pro-B-type natriuretic peptide levels compared with those in the reference group. In the present study, we also found that patients with a $\triangle \mathrm{MHSBP}$ of $<-5 \mathrm{~mm} \mathrm{Hg}$ were more likely to have a history of cerebro- or cardiovascular disease as well as complications of diabetes mellitus or chronic kidney disease, which may have influenced their prognosis. Among the five serial CBP measurements, Velasco et al. ${ }^{26}$ reported that the first CBP reading had prognostic significance. Participants were categorized into four groups based on the first $\mathrm{CBP}$ and the averaged third to fifth CBP readings: 1 , normal 1st CBP and normal averaged 3rd to 5th CBP; 2, high 1st CBP and normal averaged 3rd to 5th CBP; 3 , normal 1 st CBP and high averaged 3rd to 5th $\mathrm{CBP}$ and 4, high 1st $\mathrm{CBP}$ and high averaged 3rd to 5th CBP in their study. They found a higher cumulative incidence for cardiovascular events in participants with high 1st CBP and normal averaged 3rd to 5th CBP compared with those with normal 1st CBP and normal averaged 3rd to 5th CBP during the 9.4 years of follow-up. They also reported that $\Delta 1$ st CBP and the averaged 3rd to 5th CBP readings were associated with cardiovascular events independent of the averaged 3rd to 5th CBP readings.

Our findings demonstrate a higher cardiovascular risk in patients with a large $\triangle \mathrm{MHSBP}$ compared with patients with a small $\triangle \mathrm{MHSBP}$. Specifically, those with a higher averaged MHSBP indicated the importance of using the averaged HBP values and the differences between the first and second HBP measurements as predictors of cardiovascular events.

\section{Limitations}

The present study has some limitations. First, the HONEST study is an observational study designed to reflect real-world clinical practice. Therefore, patients were not blinded to their treatment and there was no control group. No BP targets were specified, and BP control was used at the discretion of the attending physicians.

Second, the present study enrolled patients who had already owned cuff oscillometric devices to measure their HBP, and no specific types of device were used. However, HBP-measuring devices available in Japan have been validated and approved by the Ministry of Health, Labor and Welfare of Japan and are in accordance with United States (Association for the Advancement of Medical Instrumentation) ${ }^{16}$ or European standards. ${ }^{17}$ Therefore, we believe the influence due to the use of different devices is limited.
Third, in the present study, only 2 days of HBP values were used for each measurement point to reduce the burden on patients and physicians in collecting these measurements. To minimize the potential for selection bias, detailed procedures for measuring and reporting HBP values were defined in the study protocol. Nevertheless, the possibility of biased reporting cannot be excluded completely. Although it is generally accepted that potential selection bias is reduced with an increase in sample size, the present study is limited by the nature of its design (observational study).

Finally, the mean follow-up period of the present study was relatively short (2.02 years).

\section{CONCLUSION}

First, second and averaged MHSBP during the follow-up period have similar prognostic values. Patients with a large $\triangle \mathrm{MHSBP}$ have a worse prognosis than those with a small $\triangle$ MHSBP. Furthermore, cardiovascular risk is particularly increased in patients with both a large $\triangle \mathrm{MHSBP}$ and a higher averaged MHSBP. Therefore, we recommend that the HBP be measured twice on one occasion and that the average value of the two measurements be used to monitor patients, with particular attention paid to those with a large $\triangle$ MHSBP.

\section{CONFLICT OF INTEREST}

Drs Saito, Kario, Kushiro, Teramukai and Shimada have received honoraria from Daiichi Sankyo. Yaginuma, Mori and Okuda are employees of Daiichi Sankyo.

\section{ACKNOWLEDGEMENTS}

We gratefully acknowledge the numerous study investigators, fellows, nurses and research coordinators who participated in the HONEST study. The study was funded by Daiichi Sankyo. The sponsor was involved in the design, conduct, analysis and reporting of the study. All authors contributed to writing or critically reviewing the manuscript, and approved the final version of the manuscript for submission.

1 Kario K, Saito I, Kushiro T, Teramukai S, Ishikawa Y, Mori Y, Kobayashi F, Shimada K. Home blood pressure and cardiovascular outcomes in patients during antihypertensive therapy: primary results of HONEST, a large-scale prospective, real-world observational study. Hypertension 2014; 64: 989-996.

2 Ohkubo T, Imai Y, Tsuji I, Nagai K, Kato J, Kikuchi N, Nishiyama A, Aihara A, Sekino M, Kikuya M, Ito S, Satoh H, Hisamichi S. Home blood pressure measurement has a stronger 
predictive power for mortality than does screening blood pressure measurement: a population-based observation in Ohasama, Japan. J Hypertens 1998; 16: 971-975.

3 Asayama K, Ohkubo T, Sato A, Hara A, Obara T, Yasui D, Metoki H, Inoue R, Kikuya M, Hashimoto J, Hoshi H, Satoh H, Imai Y. Proposal of a risk-stratification system for the Japanese population based on blood pressure levels: the Ohasama study. Hypertens Res 2008; 31: 1315-1322.

4 Niiranen TJ, Hänninen MR, Johansson J, Reunanen A, Jula AM. Home-measured blood pressure is a stronger predictor of cardiovascular risk than office blood pressure: the Finn-Home study. Hypertension 2010; 55: 1346-1351.

5 Bobrie G, Chatellier G, Genes N, Clerson P, Vaur L, Vaisse B, Menard J, Mallion JM. Cardiovascular prognosis of 'masked hypertension' detected by blood pressure selfmeasurement in elderly treated hypertensive patients. JAMA 2004; 291: 1342-1349.

6 Sega R, Facchetti R, Bombelli M, Cesana G, Corrao G, Grassi G, Mancia G. Prognostic value of ambulatory and home blood pressures compared with office blood pressure in the general population: follow-up results from the Pressioni Arteriose Monitorate e Loro Associazioni (PAMELA) study. Circulation 2005; 111: 1777-1783.

7 Asayama K, Wei FF, Liu YP, Hara A, Gu YM, Schutte R, Li Y, Thijs L, Staessen JA. Does blood pressure variability contribute to risk stratification? Methodological issues and a review of outcome studies based on home blood pressure. Hypertens Res 2015; 38: 97-101.

8 Shimamoto K, Ando K, Fujita T, Hasebe N, Higaki J, Horiuchi M, Imai Y, Imaizumi T, Ishimitsu $\mathrm{T}$, Ito $\mathrm{M}$, Ito $\mathrm{S}$, Itoh $\mathrm{H}$, Iwao $\mathrm{H}$, Kai H, Kario $\mathrm{K}$, Kashihara N, Kawano $\mathrm{Y}$, Kim-Mitsuyama S, Kimura G, Kohara K, Komuro I, Kumagai H, Matsuura H, Miura K, Morishita R, Naruse M, Node K, Ohya Y, Rakugi H, Saito I, Saitoh S, Shimada K, Shimosawa T, Suzuki H, Tamura K, Tanahashi N, Tsuchihashi T, Uchiyama M, Ueda S, Umemura S. Japanese Society of Hypertension Committee for Guidelines for the Management of Hypertension. The Japanese Society of Hypertension Guidelines for the Management of Hypertension (JSH 2014). Hypertens Res 2014; 37: 253-390.

9 Mancia G, Fagard R, Narkiewicz K, Redón J, Zanchetti A, Böhm M, Christiaens T, Cifkova R, De Backer G, Dominiczak A, Galderisi M, Grobbee DE, Jaarsma T, Kirchhof P, Kjeldsen SE, Laurent S, Manolis AJ, Nilsson PM, Ruilope LM, Schmieder RE, Sirnes PA, Sleight P, Viigimaa M, Waeber B, Zannad F. Task Force Members. 2013 ESH/ESC Guidelines for the management of arterial hypertension: the Task Force for the management of arterial hypertension of the European Society of Hypertension (ESH) and of the European Society of Cardiology (ESC). J Hypertens 2013; 31: 1281-1357.

10 Niiranen TJ, Johansson JK, Reunanen A, Jula AM. Optimal schedule for home blood pressure measurement based on prognostic data: the Finn-Home Study. Hypertension 2011; 57: 1081-1086.

11 Stergiou GS, Nasothimiou EG, Kalogeropoulos PG, Pantazis N, Baibas NM. The optimal home blood pressure monitoring schedule based on the Didima outcome study. J Hum Hypertens 2010; 24: 158-164.

12 Saito I, Kario K, Kushiro T, Teramukai S, Zenimura N, Hiramatsu K, Kobayashi F, Shimada K. Rationale, study design, baseline characteristics and blood pressure at 16 weeks in the HONEST Study. Hypertens Res 2013; 36: 177-182.

13 Kawabe H, Kanda T, Hirose H, Saito I. Variability of home blood pressure measurements between first and second measurements on one occasion, and factors related to variability. Clin Exp Hypertens 2012; 34: 237-242.

14 Shimada K, Kario K, Kushiro T, Teramukai S, Ishikawa Y, Kobayashi F, Saito I. Differences between clinic blood pressure and morning home blood pressure, as shown by Bland-Altman plots, in a large observational study (HONEST study). Hypertens Res 2015; 38: 876-882.

15 Kario K, Ishikawa J, Pickering TG, Hoshide S, Eguchi K, Morinari M, Hoshide Y, Kuroda T, Shimada K. Morning hypertension: the strongest independent risk factor for stroke in elderly hypertensive patients. Hypertens Res 2006; 29: 581-587.

16 Association for the Advancement of Medical Instrumentation. American National Standard. Electronic or Automated Sphygmomanometers, ANSI/AAMI SP 10-1992. AAMI: Arlington, VA, 1993, p 40.
17 European Committee for Standardization. Non-invasive Sphygmomanometers. Part 3. Supplementary Requirements for Electromechanical Blood Pressure Measuring Systems, British Standard BS EN 1060-3: 1997. European Standard EN 1060-3 1997. ECS: Brussels, Belgium, 1997.

18 Ogihara T, Kikuchi K, Matsuoka H, Fujita T, Higaki J, Horiuchi M, Imai Y, Imaizumi T, Ito S, Iwao H, Kario K, Kawano Y, Kim-Mitsuyama S, Kimura G, Matsubara H, Matsuura H, Naruse M, Saito I, Shimada K, Shimamoto K, Suzuki H, Takishita S, Tanahashi N, Tsuchihashi T, Uchiyama M, Ueda S, Ueshima H, Umemura S, Ishimitsu T, Rakugi H. Japanese Society of Hypertension Committee. The Japanese Society of Hypertension Guidelines for the Management of Hypertension (JSH 2009). Hypertens Res 2009; 32: 3-107.

19 Li Y, Thijs L, Hansen TW, Kikuya M, Boggia J, Richart T, Metoki H, Ohkubo T, Torp-Pedersen C, Kuznetsova T, Stolarz-Skrzypek K, Tikhonoff V, Malyutina S, Casiglia E, Nikitin Y, Sandoya E, Kawecka-Jaszcz K, Ibsen H, Imai Y, Wang J, Staessen JA. International Database on Ambulatory Blood Pressure Monitoring in Relation to Cardiovascular Outcomes Investigators. Prognostic value of the morning blood pressure surge in 5645 subjects from 8 populations. Hypertension 2010; 55: 1040-1048.

20 Kario K, Pickering TG, Matsuo T, Hoshide S, Schwartz JE, Shimada K. Stroke prognosis and abnormal nocturnal blood pressure falls in older hypertensives. Hypertension 2001; 38: 852-857.

21 Johansson JK, Niiranen TJ, Puukka PJ, Jula AM. Prognostic value of the variability in home-measured blood pressure and heart rate: the Finn-Home Study. Hypertension 2012; 59: 212-218.

22 Stergiou GS, Ntineri A, Kollias A, Ohkubo T, Imai Y, Parati G. Blood pressure variability assessed by home measurements: a systematic review. Hypertens Res 2014; 37: 565-572.

23 Rothwell PM, Howard SC, Dolan E, O'Brien E, Dobson JE, Dahlöf B, Sever PS, Poulter NR. Prognostic significance of visit-to-visit variability, maximum systolic blood pressure, and episodic hypertension. Lancet 2010; 375: 895-905.

24 Eto M, Toba K, Akishita M, Kozaki K, Watanabe T, Kim S, Hashimoto M, Ako J, lijima $\mathrm{K}$, Sudoh N, Yoshizumi M, Ouchi Y. Impact of blood pressure variability on cardiovascular events in elderly patients with hypertension. Hypertens Res 2005; 28 : $1-7$.

25 Shibasaki S, Hoshide S, Eguchi K, Ishikawa J, Kario K. The Japan Morning Surge-Home Blood Pressure (J-HOP) Study Group. Increase trend in home blood pressure on a single occasion is associated with B-type natriuretic peptide and the estimated glomerular filtration rate. Am J Hypertens 2015; 28: 1098-1105.

26 Velasco A, Ayers C, Das SR, de Lemos JA, Khera A, Victor RG, Kaplan NM, Vongpatanasin W. Target organ complications and prognostic significance of alerting reaction: analysis from the Dallas heart study. J Hypertens 2016; 34: 226-234.

(c) (i) (2) (2) This work is licensed under a Creative Commons At NC SA Atribution-NonCommercial-ShareAlike 4.0 International License. The images or other third party material in this article are included in the article's Creative Commons license, unless indicated otherwise in the credit line; if the material is not included under the Creative Commons license, users will need to obtain permission from the license holder to reproduce the material. To view a copy of this license, visit http://creativecommons.org/licenses/bync-sa/4.0/

(C) The Author(s) 2016 